\title{
Slow magneto-acoustic waves in simulations of a solar plage region carry enough energy to heat the chromosphere
}

\author{
N. Yadav ${ }^{1, \star} \odot$, R. H. Cameron ${ }^{1}$, and S. K. Solanki ${ }^{1,2}$ \\ ${ }^{1}$ Max-Planck-Institut für Sonnensystemforschung, Justus-von-Liebig-Weg 3, 37077 Göttingen, Germany \\ e-mail: yadavn@mps .mpg.de, solanki@mps .mpg.de \\ 2 School of Space Research, Kyung Hee University, Yongin, Gyeonggi 446-701, Republic of Korea
}

Received 13 November 2020 / Accepted 1 May 2021

\begin{abstract}
Aims. We study the properties of slow magneto-acoustic waves that are naturally excited as a result of turbulent convection and we investigate their role in the energy balance of a plage region using three dimensional radiation magnetohydrodynamic simulations.

Methods. To follow slow magneto-acoustic waves traveling along the magnetic field lines, we selected 25 seed locations inside a strong magnetic element and tracked the associated magnetic field lines both in space and time. We calculate the longitudinal component (i.e., parallel to the field) of velocity at each grid point along the field line and compute the temporal power spectra at various heights above the mean solar surface. Additionally, the horizontally-averaged (over the whole domain) frequency power spectra for both longitudinal and vertical (i.e., the component perpendicular to the surface) components of velocity are calculated using time series at fixed locations. To compare our results with the observations, we degrade the simulation data with Gaussian kernels having a full width at half maxium of $100 \mathrm{~km}$ and $200 \mathrm{~km}$ and calculate the horizontally-averaged power spectra for the vertical component of velocity using time series at fixed locations.

Results. The power spectra of the longitudinal component of velocity, averaged over 25 field lines in the core of a kG magnetic flux concentration reveal that the dominant period of oscillations shifts from $\sim 6.5 \mathrm{~min}$ in the photosphere to $\sim 4 \mathrm{~min}$ in the chromosphere. This behavior is consistent with earlier studies that were restricted to vertically propagating waves. At the same time, the velocity power spectra, averaged horizontally over the whole domain, show that low frequency waves $(\sim 6.5$ min period $)$ may reach well into the chromosphere. In addition, the power spectra at high frequencies follow a power law with an exponent close to $-5 / 3$, suggestive of turbulent excitation. Moreover, waves with frequencies above $5 \mathrm{mHz}$ propagating along different field lines are found to be out of phase with each other, even within a single magnetic concentration. The horizontally-averaged power spectra of the vertical component of velocity at various effective resolutions show that the observed acoustic wave energy fluxes are underestimated by a factor of three, even if determined from observations carried out at a high spatial resolution of $200 \mathrm{~km}$. Since the waves propagate along the non-vertical field lines, measuring the velocity component along the line-of-sight, rather than along the field, contributes significantly to this underestimation. Moreover, this underestimation of the energy flux indirectly indicates the importance of high-frequency waves that are shown to have a smaller spatial coherence and are thus more strongly influenced by the spatial averaging effect compared to low-frequency waves.

Conclusions. Inside a plage region, there is on average a significant fraction of low frequency waves leaking into the chromosphere due to inclined magnetic field lines. Our results show that longitudinal waves carry (just) enough energy to heat the chromosphere in the solar plage. However, phase differences between waves traveling along different field lines within a single magnetic concentration can lead to underestimations of the wave energy flux due to averaging effects in degraded simulation data and, similarly, in observations with lower spatial resolution. We find that current observations (with spatial resolution around $200 \mathrm{~km}$ ) underestimate the energy flux by roughly a factor of three - or more if the observations are carried out at a lower spatial resolution. We expect that even at a very high resolution, which is expected with the next generation of telescopes such as DKIST and the EST, less than half, on average, of the energy flux carried by such waves will be detected if only the line-of-sight component of the velocity is measured.
\end{abstract}

Key words. Sun: chromosphere - Sun: faculae, plages - Sun: magnetic fields - methods: numerical

\section{Introduction}

The Solar surface is populated with magnetic structures existing at various spatial scales, such as large sunspots, intermediatesized pores and plage magnetic features, as well as smallscale internetwork magnetic bright points, etc. The magnetic field concentrated in such features plays an important role in the wave energy transport from the turbulent convection

\footnotetext{
* Current address: Centre for mathematical Plasma Astrophysics, Department of Mathematics, KU Leuven, Celestijnenlaan 200B, 3001 Leuven, Belgium.
}

zone to the upper layers of the solar atmosphere (Solanki et al. 2006; Mathioudakis et al. 2013). Beneath the solar surface, acoustic p-modes are generated by turbulent convection (Goldreich \& Keeley 1977; Nordlund \& Stein 2001). At the layer where the sound speed is equal to the Alfvén velocity, these incoming acoustic waves or p-modes are partly "converted" into fast waves of a magnetic nature and partly "transmitted" as slow waves without any change to their acoustic nature (Cally 2007; Khomenko \& Cally 2012). Propagating upward through the solar atmosphere, the amplitude of acoustic waves increases because of the rapid decrease in density with increasing height and are dissipated by shock formation (Vecchio et al. 2009). 
Decades ago, Biermann (1948) and Schwarzschild (1948) suggested this acoustic wave-based heating mechanism as a plausible means of heating the non-magnetic chromosphere of the quiet Sun. Their proposal was followed by several numerical and observational studies (Ulmschneider et al. 2005; Praderie \& Thomas 1976) and was long considered an important heating source in the lower chromosphere. Nonetheless, using the observations from the Transition Region and Coronal Explorer (TRACE; Handy et al. 1999), Fossum \& Carlsson (2005a,b, 2006) concluded that the wave energy flux of highfrequency acoustic waves is insufficient to account for the radiative losses in the non-magnetic solar chromosphere and, thus, cannot constitute the dominant heating mechanism. Later, Cuntz et al. (2007) criticized these results and suggested that high-frequency acoustic waves can indeed be sufficient to heat the nonmagnetic solar chromosphere.

With increasing observational capabilities, the interest in investigating the role of high-frequency $(f>10 \mathrm{mHz})$ magnetoacoustic waves in chromospheric heating was revived. Using observations from the Göttingen Fabry-Perot spectrometer (FPI) at the German Vacuum Tower Telescope, Bello González et al. $(2009,2010 \mathrm{a})$ found significant energy flux $\left(\sim 3000 \mathrm{~W} \mathrm{~m}^{-2}\right)$ in frequencies above the acoustic cutoff frequency $(\sim 5.2 \mathrm{mHz})$. Then, by analyzing the observations recorded by the Imaging Magnetograph eXperiment (IMaX) spectropolarimeter onboard the SUNRISE balloon-based observatory (Solanki et al. 2010; Barthol et al. 2011; Gandorfer et al. 2011; Berkefeld et al. 2011), Bello González et al. (2010b) gave strength to the idea that acoustic waves with periods shorter than the acoustic cutoffperiod can significantly contribute to the heating of the solar chromosphere. However, they could not conclusively verify that the acoustic wave energy flux is sufficient to compensate for the radiative losses of the non-magnetic quiet solar chromosphere.

More recently, using high spatial and temporal resolution data from the SUNRISE Filter Imager (SuFI, Gandorfer et al. 2011), Jafarzadeh et al. (2017) investigated the propagation of acoustic waves in the lower solar atmosphere by analyzing both the horizontal-displacement oscillations of magnetic bright points and their intensity perturbations. They observed upward propagating compressible and incompressible (kink) waves at high frequencies (up to $30 \mathrm{mHz}$ ). Previously, using numerical modeling, Hasan et al. (2005) had proposed the existence of short-period magneto-acoustic waves ( $20 \mathrm{~s})$ and suggested that they are excited due to the random displacements of magnetic field lines caused by intergranular turbulence. Lawrence et al. (2011) analyzed the data recorded with the Rapid Oscillations in the Solar Atmosphere (ROSA) instrument and supported the idea of high-frequency acoustic wave excitation due to photospheric turbulence and showed that spectral power in the $28-326 \mathrm{mHz}$ range follows a power law with exponent $-1.21 \pm 0.02$, consistent with the presence of turbulent motions. Overall, there have been numerous observational and simulation studies over the last half-century to quantify the role of slow magneto-acoustic waves in the heating of solar chromosphere. However, possibly due to the limited spatial resolution and cadence from which observations suffer, these waves have never been found to carry sufficient energy flux to explain chromospheric heating.

Most of the aforementioned wave studies correspond to quiet Sun and magnetic bright points, however, acoustic waves play an important role in heating the magnetic chromosphere as well (Lites et al. 1993; Kalkofen 2007). The active region chromosphere is filled with small-scale, short-lived jet-like features called fibrils (e.g., Pietarila et al. 2009) that are believed to make a non-negligible contribution to the heating of the solar chromosphere (Withbroe 1983; De Pontieu et al. 2004). These fibrils (spicules when located on the solar limb) are driven by magneto-acoustic shocks formed by leakage of upward propagating slow magneto-acoustic waves from the photosphere (Hansteen et al. 2006; De Pontieu et al. 2007).

Moreover, acoustic oscillations in the photosphere do not always display the same periods as in the chromosphere. For example, five-min oscillations are not observed in the chromosphere of sunspots (Bhatnagar \& Tanaka 1972; Bogdan 2000; Bogdan \& Judge 2006 and references therein). Lites (1986) showed that regions with high oscillatory power in the 3-min band are uncorrelated with the regions of high oscillatory power in the 5-min band within the umbrae, which suggests that the 5-min oscillations in the photosphere do not excite the 3-min oscillations in the chromosphere of sunspots. However, the high-frequency peaks of the photospheric power spectra are often correlated with the peaks in the power spectra of chromospheric oscillations (Lites \& Thomas 1985; Centeno et al. 2006). Centeno et al. (2006) performed phase diagnostics of the chromospheric and the photospheric oscillations and suggested that the 3-min oscillations observed in the chromosphere come from the photosphere by means of linear wave propagation, rather than from nonlinear interaction of 5-min modes. Using time series observations of various spectral lines originating at different heights in the solar atmosphere, Centeno et al. (2009) investigated the propagation of magneto-acoustic waves in different magnetic regions on the Sun. They showed that photospheric oscillations have similar characteristics in all the regions, whereas chromosopheric oscillations depend on their magnetic properties (e.g., strength and inclination of magnetic field through which they propagate). Power spectra of chromospheric oscillations in the plage regions are shown to exhibit a dominant peak in the 5-min band in contrast to sunspots and quiet Sun (Bhatnagar \& Tanaka 1972; Centeno et al. 2009). Lites et al. (1993) analyzed and compared the solar oscillations between the chromospheric network and internetwork regions using $1 \mathrm{~h}$ sequence of spectrograms of a quiet Sun region. They found internetwork chromospheric velocity power spectrum is dominated by high-frequency $(\sim 5 \mathrm{mHz})$ oscillations while power spectra in network regions are dominated by lowfrequency oscillations with periods of 5-20 min. Similar results are recently obtained by Rajaguru et al. (2019) using Helioseismic and Magnetic Imager and lower-atmospheric UV emission maps in the 170 and $160 \mathrm{~nm}$ channels of the Atmospheric Imaging Assembly, both onboard the Solar Dynamics Observatory of NASA.

The shift of the dominant wave period from 5-min in the photospheric oscillations to 3-min in the chromospheric oscillations is often attributed to the cut-off period of the temperature minimum (Michalitsanos 1973; Bel \& Leroy 1977; Fleck \& Schmitz 1991; Verth \& Jess 2016; McIntosh \& Jefferies 2006; Khomenko \& Collados 2015; Chae et al. 2017). The effective cut-off period may, however, increase due to inclination of the magnetic field relative to the vertical (Schwartz \& Bel 1984; De Pontieu et al. 2004, 2005) or due to departure from adiabaticity (Khomenko et al. 2008; Felipe \& Sangeetha 2020), resulting in the propagation of waves with periods of more than 3-min. de Wijn et al. (2009) showed that waves with 3-min periods propagate in the central facular chromosphere where magnetic field is more vertical while 5-min waves propagate at the peripheral regions where the magnetic field is both inclined and expanding.

In this paper, we investigate the properties of slow magnetoacoustic waves in radiation magnetohydrodynamic (MHD) 


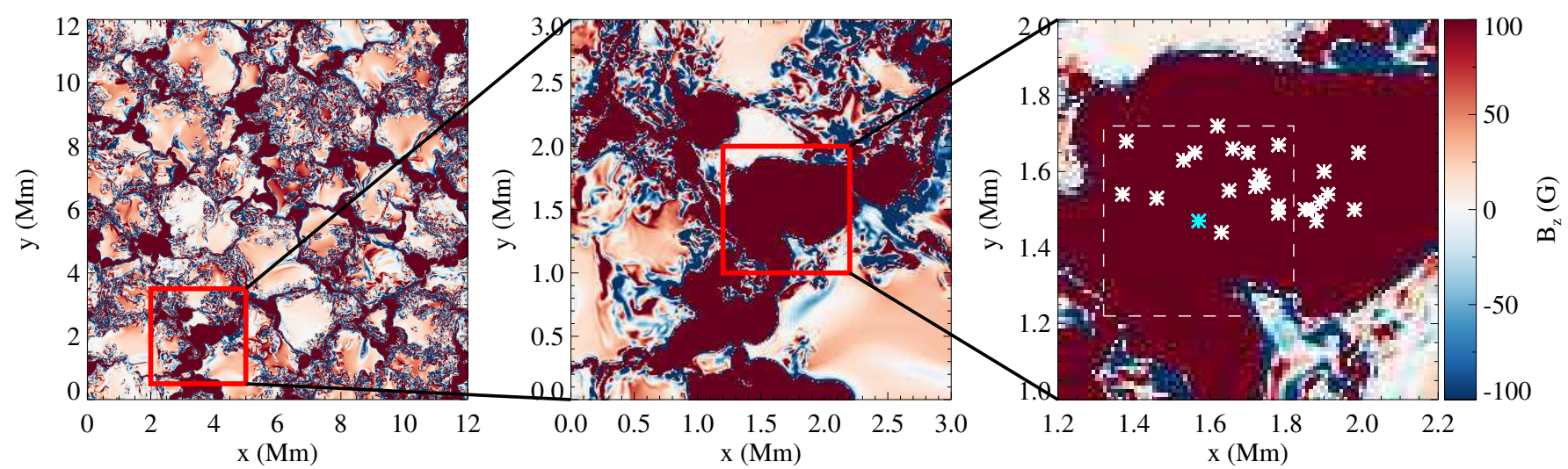

Fig. 1. 2D maps of the vertical component of the magnetic field vector and initial locations of seed points. Left: $2 \mathrm{D}$ map of the vertical component of the magnetic field vector at $z=0$ layer (corresponding to average $\tau=1$ layer) covering the whole horizontal extent of the simulation domain (saturated at $\pm 100 \mathrm{G}$ ), middle: blowup displaying the sub-region (red box in the left panel) selected for magneto-acoustic wave analysis. Right: blowup displaying the seed locations (see main text for details). The dashed square in the rightmost panel covers the region shown in Fig. 10.

simulations of a plage region by tracking magnetic field lines in both space and time. We compare the temporal power spectra of longitudinal velocity averaged over 25 field lines inside a strong magnetic element with the horizontally-averaged (over the whole domain) power spectra. To make comparisons with the observations, we degraded our simulation data and calculate the power spectra of the vertical component of velocity and compared it to the power spectra obtained with full resolution data. In addition, we estimated the acoustic energy flux associated with slow magneto-acoustic waves for the full resolution and for the degraded data as well.

This paper is organized as follows: Sect. 2 comprises the details of numerical simulations and methodology used for field line tracking. The results are presented and discussed in Sect. 3. Our summary and concluding remarks are given in Sect. 4.

\section{Methodology}

\subsection{Simulation setup}

We used the three dimensional (3D) radiation-MHD code MURaM (Vögler et al. 2005; Rempel 2014, 2017) to simulate a unipolar plage region. The basic ingredients of the MURaM code are the MHD equations that are solved using a fourth-order finite difference scheme along with a short characteristic radiative transfer scheme. This code takes into account most of the essential physics required to simulate the upper convection zone where acoustic waves are excited and the lower solar atmosphere where magneto-acoustic waves transform into shocks. The simulation domain is $12 \mathrm{Mm} \times 12 \mathrm{Mm} \times 4 \mathrm{Mm}$ (with the third dimension referring to the vertical $z$-direction) with the top boundary located at $2500 \mathrm{~km}$ above the average $\tau=1$ layer (continuum formation height). The simulation domain is resolved by $1200 \times 1200 \times 400$ grid points with a grid spacing of $10 \mathrm{~km}$ in all three directions. The simulation domain is periodic in the horizontal directions with an open bottom boundary and closed top boundary. For the magnetic field, we used a vertical magnetic field as the top boundary condition.

To simulate a unipolar plage region, we ran the hydrodynamic simulations for almost $2 \mathrm{~h}$ to reach a statistically relaxed state and then introduced a uniform vertical magnetic field of 200 $\mathrm{G}$ and ran it again for $1.2 \mathrm{~h}$, so that the system again reaches a statistically stationary state. Then, to investigate wave properties, we collect a sequence of $25 \mathrm{~min}$ at a $1 \mathrm{~s}$ cadence for a sub-region of $3 \mathrm{Mm} \times 3 \mathrm{Mm} \times 3 \mathrm{Mm}$ that extends from $500 \mathrm{~km}$ below the mean solar surface to $2.5 \mathrm{Mm}$ above the mean solar surface. We chose a smaller sub-region due to disk space and memory constraints but did not compromise on temporal cadence and spatial resolution for the purpose of capturing high-frequency waves. Figure 1(left panel) displays a map of the vertical component of the magnetic field at the mean solar surface $(z=0)$ at the beginning of the sequence. The selected sub-region chosen for the wave study corresponds to the red box in the left panel and is shown on an enlarged scale in the middle panel of Fig. 1. The right panel of this figure displays the region where we placed the seed points (shown as asterisks) to perform the wave analysis (described in the next subsection).

\subsection{Wave analysis: Field line tracking}

The solar photosphere is highly turbulent and dynamic. Granular buffeting and turbulent plasma motions excite a wide variety of waves over a large frequency range that may dissipate and heat the solar atmosphere. Many of the previous numerical simulations invoke wave excitation sources, for instance, transverse and torsional drivers mimicking the granular buffeting and photospheric vortex motions, respectively (Vigeesh et al. 2012). These drivers perturb the photospheric foot-points of magnetic flux tubes, exciting perturbations within them, which then travel to higher layers along the magnetic field lines in the form of MHD waves. However, there can be many other sources that may excite MHD waves, such as granular buffeting, intergranular turbulence (Hasan et al. 2000; Chitta et al. 2012), intermittent impulsive events (Jafarzadeh et al. 2013), magnetic pumping (Kato et al. 2011, 2016), mode conversion and transmission (Cally 2007; Khomenko \& Cally 2012), the interaction of the magnetic elements with the convective flows (van Ballegooijen et al. 2011), etc. Therefore, it is crucial to include these additional waveexcitation sources in numerical models in as natural a way as possible, namely, without having to prescribe them. If they are included roughly as they occur on the Sun, we would be able to quantify the net energy flux being carried by these waves and their importance in heating the chromosphere.

With this motivation, we performed numerical simulations of a plage region and investigate the propagation of magnetoacoustic waves that are naturally excited due to turbulent convection. Therefore, unlike in most earlier studies, we do not prescribe the external perturbation of the magnetic features, so 


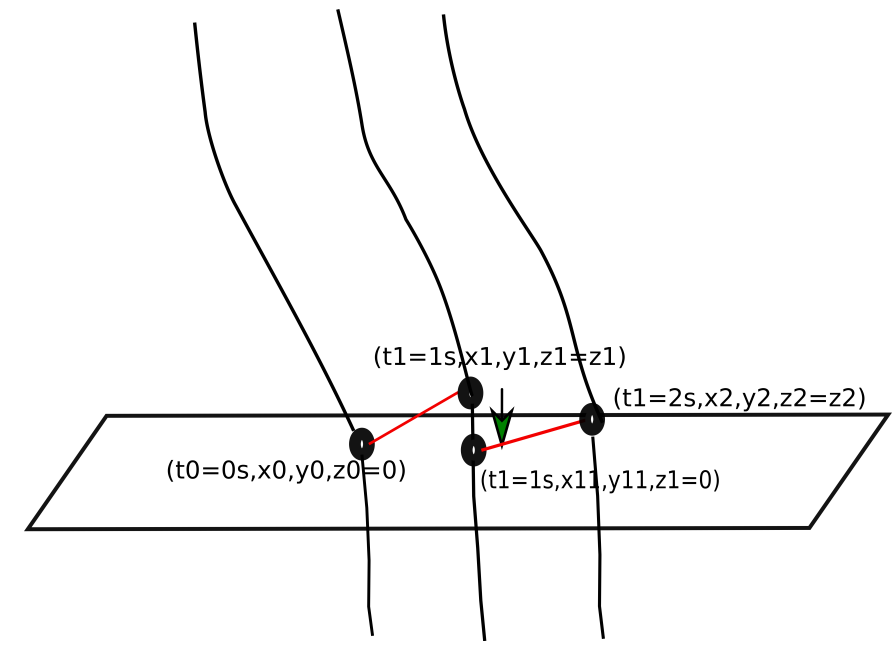

Fig. 2. Scheme used to track a single magnetic field line over time.

that we expect that various wavemodes are excited at amplitudes similar to those also present on the real Sun. These excited slow magneto-acoustic waves travel along the magnetic field lines through the solar atmosphere. Given that the magnetic field is advected by turbulent granular motions, it is important to discern the temporal evolution of the magnetic field lines for studying waves passing along them. We selected a region hosting a wide patch of strong magnetic concentration such that magnetic field extends well into the solar atmosphere. This allows us to study the waves that could potentially propagate and impact the overlying chromosphere. To this end, we placed 100 seed points in a magnetically concentrated region at the mean solar surface. On each seed point, the magnetic field line is traced in $3 \mathrm{D}$ space using the magnetic field's vector components at $t=0$ (the beginning of time series). Thereafter, using the velocity vector data at $z=0$, we calculate the displacement of each seed point (fluid parcel) by integrating in time (here we assume that velocity does not change much in one second). Then we trace the magnetic field line from the new location of the seed point (which will not necessarily be on the $z=0$ surface) using magnetic field vector components at the next time step. Next, we use the intersections of the traced magnetic field lines at $t=1 \mathrm{~s}$ with the mean solar surface as the seed location for the next time step to follow the field line. We continue the above procedure and track the magnetic field lines over the entire course of the timesequence, namely, $25 \mathrm{~min}$. Out of 100 seed points, only 25 (displayed in the right panel of Fig. 1) stayed in the selected domain for the whole time sequence. Field lines associated with other seed points were either inclined (such that they extend beyond the selected domain at other heights) or got reconnected due to magnetic reconnection at the edges of the magnetic features or moved out of the domain in the course of $25 \mathrm{~min}$ and hence were discarded. A cartoon demonstrating this procedure for one seed point is shown in Fig. 2.

\section{Results and discussion}

As a representative case, we chose the seed point shown in the Cyan color asterisk in Fig. 1 and displayed the magnetic field line (green color) attached to it at intervals of $5 \mathrm{~min}$ in panels a-f of Fig. 3 (using VAPOR (Clyne \& Rast 2005; Clyne et al. 2007) for $3 D$ visualization). Saturated maps of the vertical component of the magnetic field strength at the mean solar surface are also displayed for each snapshot. We find that magnetic field strength at the mean solar surface evolves with time and the magnetic field line undergoes significant displacements from its initial location as its footpoint at the solar surface is advected due to the surrounding plasma motions.

The trajectory of the magnetic field line (corresponding to the selected seed point) where it cuts the $z=0$ plane is displayed in Fig. 4. Here, the color of the curve corresponds to the time elapsed since the beginning of the tracking and the axes show the spatial location. The magnetic field line travels (due to advection by plasma motions) $1.56 \mathrm{Mm}$ in total, with an average horizontal speed of $1.03 \mathrm{~km} \mathrm{~s}^{-1}$ over $25 \mathrm{~min}$. Figure 4 shows that the magnetic field line undergoes several rotations, centered roughly, for instance, at $(1.47,1.62)$ and $(1.42,1.53)$, indicating torsional motion. However, in the present paper, we mainly focus on the longitudinal motions of plasma that correspond to slow magneto-acoustic waves. The torsional motions in this simulation have been studied by Yadav et al. (2020, 2021).

We construct the height-time map of longitudinal velocity to investigate the propagation of slow magneto-acoustic wave in the simulation box. We calculate the longitudinal velocity at each vertical grid point and track the field line using the method described in Sect. 2.2. Panel a of Fig. 5 displays the heighttime map for one example. It indicates the propagation of longitudinal velocity perturbations along the magnetic field lines. Here, the vertical axis is the height normal to the mean solar surface. Since the selected region under investigation has $\mathrm{kG}$ magnetic field strength in the photosphere, the plasma beta is low, and slow magneto-acoustic waves propagate along the field lines at nearly the local sound speed. To calculate the adiabatic (or isentropic) sound speed, that is, $c_{s}=\sqrt{\gamma_{1} P / \rho}$, we use local plasma parameters and calculate the adiabatic coefficient $\left(\gamma_{1}\right)$ using Chandrasekhar's first adiabatic exponent (Chandrasekhar 1957), defined as:

$\gamma_{1}=\left(\frac{\partial \ln P}{\partial \ln \rho}\right)_{s}$.

To compute the adiabatic coefficient we used the look-up tables for the equation of state (EOS) and method of inversions of the Jacobian matrix (for details, see Appendix A.5 in Cheung 2006).

For comparison, we overplot the paths of test particles moving vertically with local sound speed as displayed by solid black lines. A dotted line is also shown that represents the height where sound speed equals the Alfvén speed. This is called the Alfvén-acoustic equipartition layer where helioseismic waves split into fast and slow magneto-acoustic branches (Cally 2007). We can see the partial transmission of incident fast acoustic waves into slow magneto-acoustic waves and partial reflection of fast magneto-acoustic waves at this layer. The slow compressive mode then travels upwards along the magnetic field lines and is converted into large-amplitude shocks due to sharp density gradients in the chromosphere. These shocks are supersonic and therefore travel faster than the local sound speed as can be seen above a height of $\sim 1 \mathrm{Mm}$. We find downflows in the layers near the top boundary due to the closed top boundary condition. Therefore, the results obtained above $\sim 1 \mathrm{Mm}$ are influenced by the top boundary condition and, hence, we do not discuss this in this paper. For comparison, we also display a height-time map of the vertical component of the velocity at the initial location of the selected seed point as it would be observed if we did not track the magnetic field line in time (Fig. 5b). We find that the map is not smooth in this case. The reason for these intermittent fluctuations is that the waves travelling in neighbouring field lines are not in phase (will be discussed later in the paper). Therefore, oscillations at a fixed location provide a false impression 
(a)

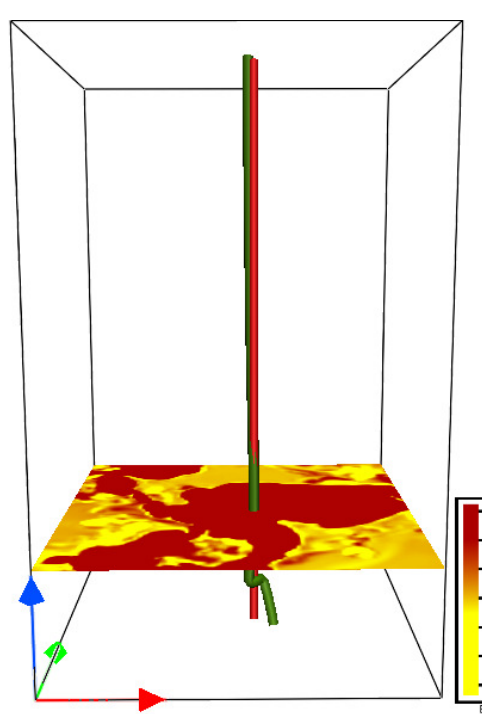

(d)

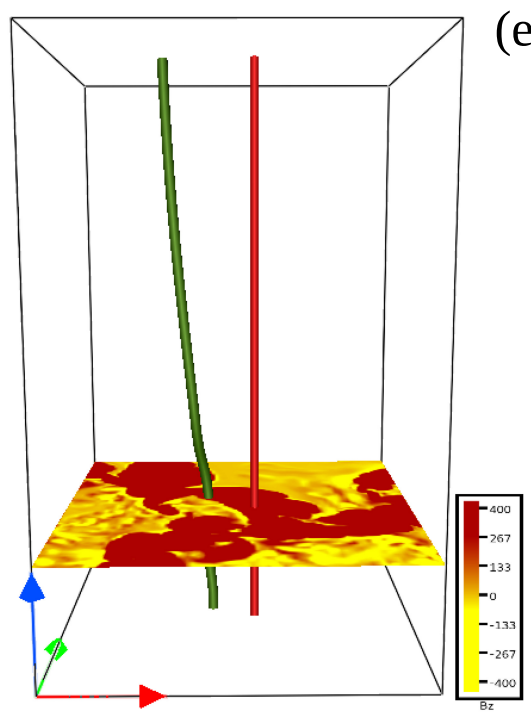

(b)

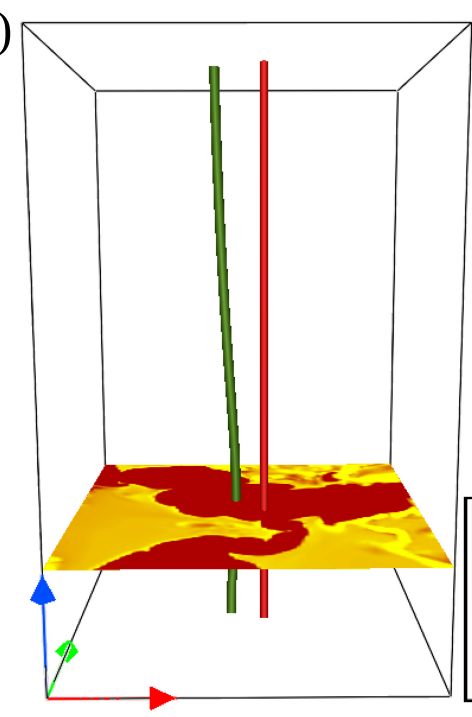

(e)

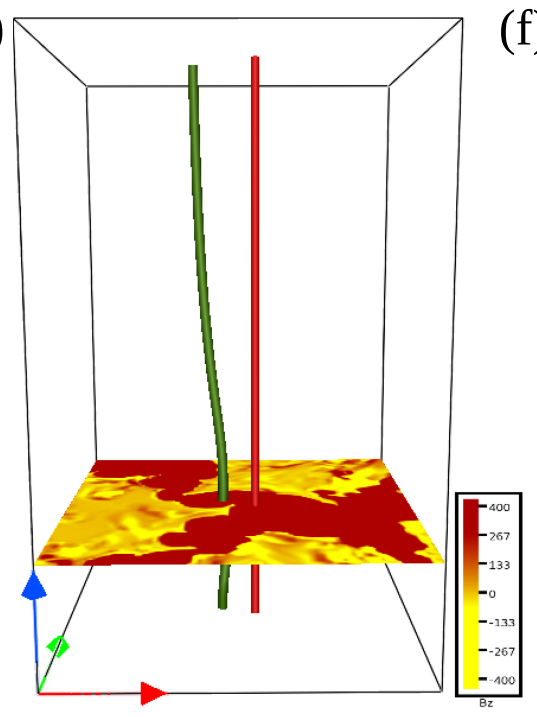

(c)

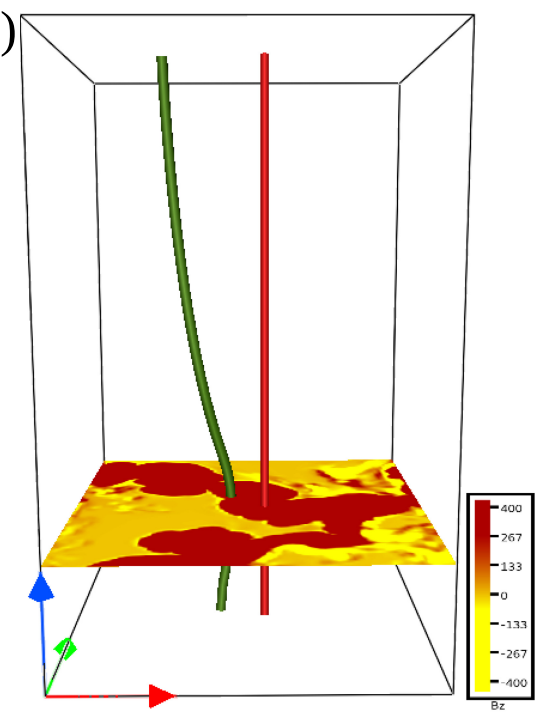

(f)

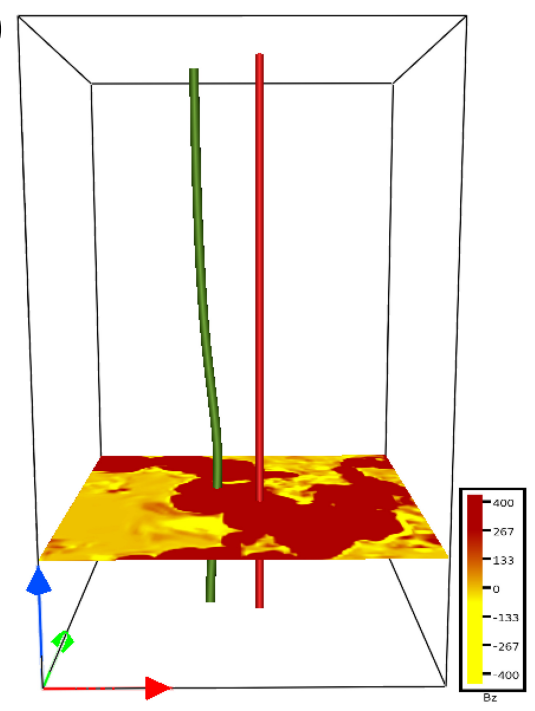

Fig. 3. Three-dimensional visualization of magnetic field line (green) corresponding to the seed point shown as a cyan asterisk in Fig. 1. The horizontal extent corresponds to the region shown in Fig. 1b. Panels $a-f$ correspond to snapshots at every 5 min starting from $t=0$ (the same instance as in Fig. 1). The red vertical line corresponds to the initial location of the seed point. Saturated maps of magnetic field strength at the mean solar surface are also shown for each temporal snapshot.

of the high-frequency component. It justifies and reinforces the importance of tracking the magnetic field lines and of measuring the longitudinal component of velocity to accurately analyze the properties of slow magneto-acoustic waves. Also, by tracking the magnetic field lines over time, we can determine the locations where linear magneto-acoustic waves become nonlinear and form magneto-acoustic shocks.

Furthermore, we determine the dominant frequencies of slow magneto-acoustic waves at various heights in the solar atmosphere. We compute height-time maps and temporal power spectra of longitudinal velocity for all the seed points displayed as white asterisks in the right panel of Fig. 1. We use the wavelet transform method as it efficiently captures short-lived features and waves (Torrence \& Compo 1998; Jess et al. 2007). In a wavelet transform, a time series of velocity or any other data is convolved with the "mother" function ('Morlet wavelet' in the present case) whose width is varied so that it captures both low and high-frequency oscillations. The output of the wavelet transform gives the time-period map which reveals the frequency/period distribution of the data at each instant of time.
Averaging over the time axis gives the global wavelet spectrum. Since we are dealing with finite time series data, errors are anticipated at the beginning and the end of the time series. To avoid these errors, the time series is padded with zeros, thus limiting the edge effects. Zero padding introduces discontinuity near the endpoints. To select the locations where wavelet power can be fully trusted, a cone of influence (COI) is calculated. The COI defines the region in which edge effects are significant. Beyond the COI, edge effects are negligible and wavelet power is reliable. A Fourier transform was also performed for comparison and verification of our wavelet transform results. However, we only present the results obtained with the wavelet transform in the paper as they are more appropriate for wave studies in the solar atmosphere, with typically short wavetrains.

Figure 6 displays the temporal power spectra of longitudinal velocity at four heights viz. $z=0, z=500 \mathrm{~km}, z=$ $1000 \mathrm{~km}$ and $z=1500 \mathrm{~km}$ in panels a-d, respectively, for the selected representative case. We display the time series of the longitudinal velocity, time-period map of the wavelet power, and time-averaged global wavelet spectrum at each height. 


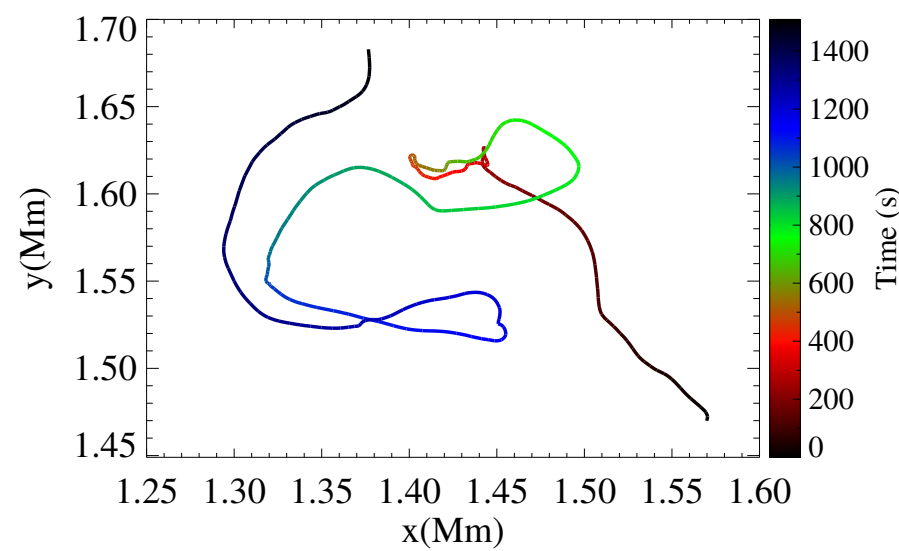

Fig. 4. Trajectory of the magnetic field line at the mean solar surface for the seed point indicated by a cyan asterisk in Fig. 1 .

The horizontal dotted line over-plotted on the global wavelet spectrum displays the maximum period for which the power can be determined by the wavelet transform. At heights of $z=0$ and $z=500 \mathrm{~km}$, the longitudinal velocity has a linear oscillatory profile whereas at $z=1000 \mathrm{~km}$ and $z=1500 \mathrm{~km}$ the waveform is no longer sinusoidal, and signs of shocks are clearly visible at these heights. In the near-surface layers, the amplitude of longitudinal velocity is smaller and the waves have periods of around 6-7 min. These high periods of velocity oscillations are possibly due to strong magnetic fields in the selected domain as the period of oscillations increases with the strength of magnetic field in solar plage (Kostik \& Khomenko 2013; Chelpanov et al. 2016). In the higher layers, wave amplitudes increase (due to density stratification), and there is more power in comparatively smaller wave-periods (higher frequencies). One reason behind the shift of peak frequency could be the frequency filtering by the upper layers that only allow waves with a frequency higher than the local cut-off frequency to pass through (Michalitsanos 1973; Bel \& Leroy 1977). It is clear from panel c and d that shocks are the dominant source for the enhanced power in lower periods or higher frequencies, as previously suggested by Vecchio et al. (2009)

To estimate the power spectra in a typical strong magnetic element with mostly vertical magnetic field lines, we take an average of frequency spectra calculated at all the seed locations (shown as white asterisks in Fig. 1). The selected 25 field lines are mostly vertical as we selected only those field lines which do not bend too strongly and stay fully in the selected domain $(3 \mathrm{Mm} \times 3 \mathrm{Mm}$ in the horizontal plane) for the whole time series. The averaged wavelet spectra, calculated by taking an average of 25 individual power spectra, are displayed in Fig. 7. At $z=0$, power distribution peaks at around $6 \mathrm{~min}$ and this peak merges with another peak at around $10.5 \mathrm{~min}$. Since our time series lasts $25 \mathrm{~min}$, results with periods beyond $9 \mathrm{~min}$ are not reliable due to the significant edge effects. Higher in the atmosphere, the distribution shifts towards lower wave-periods ( $\sim 4 \mathrm{~min})$.

However, it has been previously shown in observations that low frequency waves can leak to higher layers along the inclined magnetic field lines at the edges of strong magnetic elements in plage (Centeno et al. 2009; de Wijn et al. 2009; Rajaguru et al. 2019). To test this wave leakage in our simulations, we compute the power spectra in a similar fashion as done for Fig. 7; however, by taking an average over wavelet power spectra calculated over the whole analyzed horizontal domain $(3 \mathrm{Mm} \times 3 \mathrm{Mm})$. The resulting wavelet spectra are shown in Fig. 8. Here, we do indeed see that a significant fraction of the wavelet power at wave period $\sim 6.5 \mathrm{~min}$ is leaking to the higher atmospheric layers, which was absent in the averaged spectra over predominantly vertical field lines (Fig. 7).

Next, to calculate the frequency spectra, we convert the horizontally-averaged (over the whole analyzed domain, i.e., $3 \mathrm{Mm} \times 3 \mathrm{Mm}$ ) wavelet spectra to frequency spectra and shown these in Fig. 9. Here, black curves correspond to spectra calculated using the longitudinal component (i.e., parallel to the magnetic field) of velocity at fixed locations. Different rows correspond to different heights above the mean solar surface as mentioned in each panel. Since it is not yet possible to achieve a spatial resolution of $10 \mathrm{~km}$ with observations, we degrade our simulation data to achieve an effective resolution of $100 \mathrm{~km}$ and $200 \mathrm{~km}$. To this end, we convolve the actual simulation data with Gaussian kernels of full width at half maximum (FWHM) of $100 \mathrm{~km}$ and $200 \mathrm{~km}$, respectively. We then calculate horizontally-averaged frequency spectra of the vertical component of velocity for full resolution (red) as well for the degraded data-sets at effective resolutions of $100 \mathrm{~km}$ (blue) and $200 \mathrm{~km}$ (green), as shown in Fig. 9. A power law fit to the black curve is over-plotted as a dashed curve in the frequency range $6-25 \mathrm{mHz}$ in each panel. We find power spectra to follow a power-law of the form $a f^{-b}$ at all the heights. Here, $f$ denotes the frequency, and " $a$ " and " $b$ " are the amplitude and the exponent for the power law that are specified in each panel.

We find that power spectra have two distinct peaks centred around $2.5 \mathrm{mHz}$ and $4.5 \mathrm{mHz}$, which correspond to wave periods of $\sim 6.5 \mathrm{~min}$ and $\sim 4 \mathrm{~min}$, respectively. Comparing these spectra with the spectra shown in Fig. 7, we notice that there is a significant amount of low frequency waves $(\sim 2.5 \mathrm{mHz})$ leaking up to the higher layers that is absent in Fig. 7. This leakage of low frequency waves could be due to expansion of magnetic field lines at the edges of magnetic concentrations. This discrepancy in wave periods for vertical and non-vertical fields was previously observed and reported by de Wijn et al. (2009), who showed that low-frequency waves propagating only at the periphery of the plage. Moreover, comparing the power spectra of the velocity along the field line and in the vertical direction, we find that the power in the parallel component of the velocity is always higher than in its vertical component in the lower atmospheric layers. This is expected as slow magneto-acoustic waves travel along the field lines. However, in the upper layers where magnetic field lines are almost vertical, both red and black curves are nearly identical, implying that the longitudinal component of the velocity can be approximated by the vertical component in these layers. Here, it is worth mentioning that plasma flows in the atmospheric layers close to the computational domain's top boundary are strongly affected by the imposed closed boundary condition. Nonetheless, we showed the velocity power spectra in layers at $1 \mathrm{Mm}$ and $1.5 \mathrm{Mm}$ in Figs. 6-9 to demonstrate that low frequency waves cease to propagate into higher layers when traveling along the predominantly vertical magnetic field lines, while we find their signatures in power spectra when studying wave propagation over the whole horizontal domain.

Power spectra in the high frequency range $(6-25 \mathrm{mHz})$ approximately obey a power-law and are consistent with the observational findings by Reardon et al. (2008) who investigated the acoustic properties of the solar chromosphere in the quiet Sun using observations recorded by the Interferometric Bidimensional Spectrometer (IBIS, Cavallini 2006). They calculated power spectra of the Doppler velocity of the Ca II $854.2 \mathrm{~nm}$ line and found that the power spectrum in network regions peaks in the $3-4 \mathrm{mHz}$ range (high 5 -min power) and obeys a 


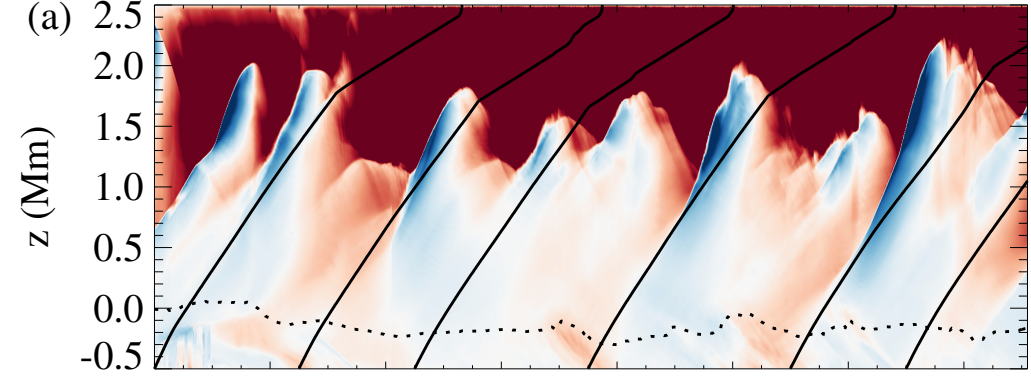

(b) 2.5
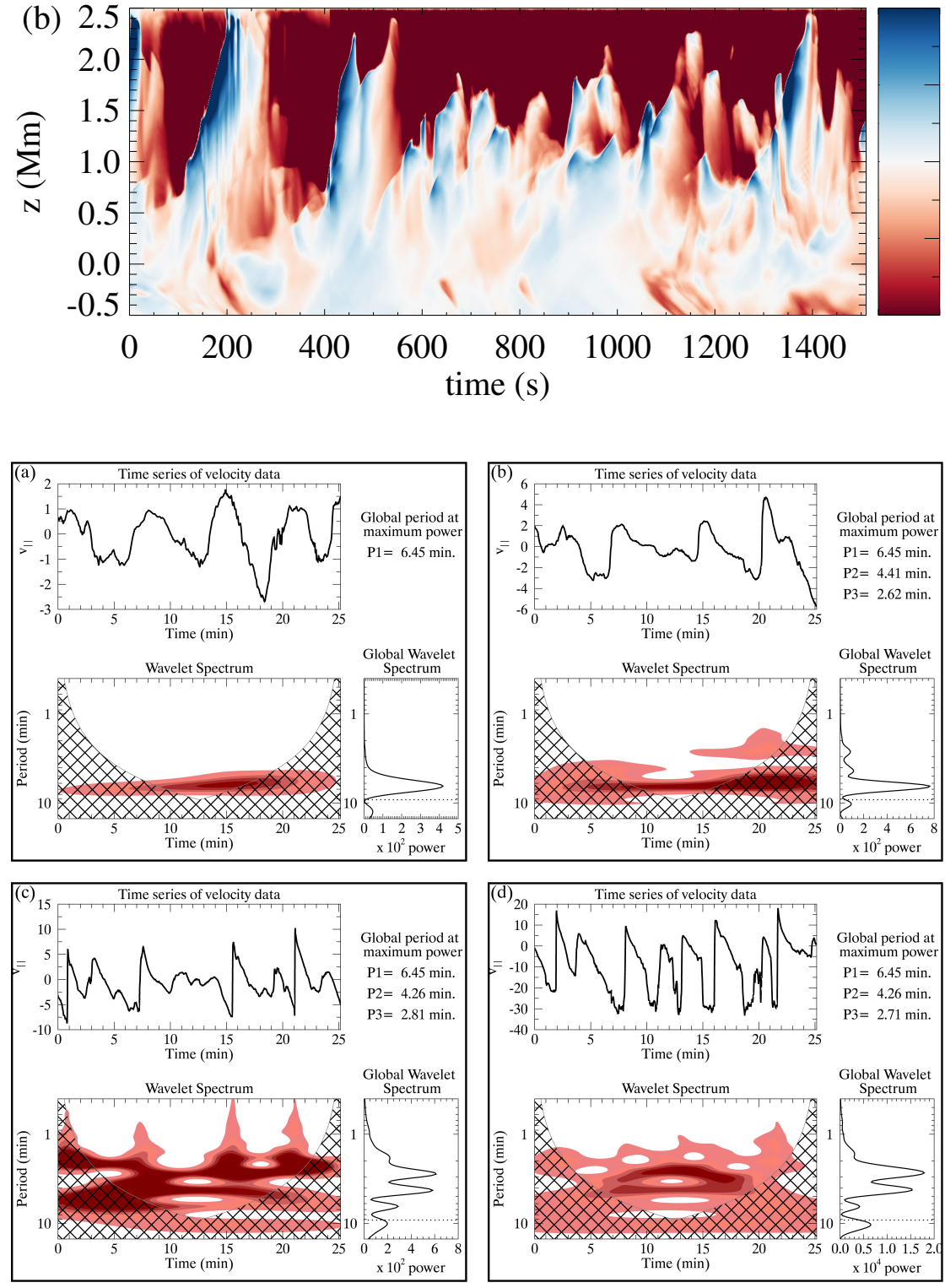

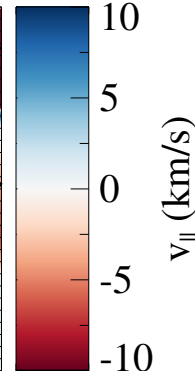

10

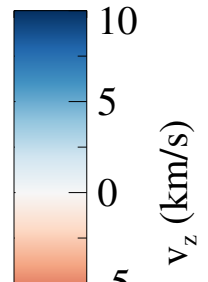

Fig. 5. Height-time map of the longitudinal and the vertical component of velocity. (a): height-time map of the component of velocity along a magnetic field line that is tracked in time using the method described in Sect. 2.2. The field line initially passing through the seed point identified by the cyan asterisk in Fig. 1 is considered here. Local sound speed curves (solid) and a curve showing the height at which $c_{s}=v_{A}$ (dotted) are also over-plotted, $(b)$ : height-time map for a fixed horizontal location i.e., initial seed location without following the magnetic field line for the seed point shown as cyan asterisk in Fig. 1. power-law with a slope of -2.4 in the range of $5-15 \mathrm{mHz}$ Although their analysis concentrated on the quiet Sun, their results for network regions can be compared with our simulation results for a plage, as both network and plage host kilogauss magnetic field concentrations and are shown to share similar velocities for equally sized magnetic patches (Buehler et al. 2019). They suggested turbulence (generated due to shocks) as the possible source of the chromospheric high-frequency power rather than propagating waves. This could be the case in our study also as there are magneto-acoustic shocks forming that may generate chromospheric turbulence. Another possible ori- gin could be the interaction between low-frequency waves that may generate high-frequency waves. The exact source of the significant power in velocities at higher frequencies is yet not clear.

Moreover, comparing the power spectra of vertical velocity obtained using actual and degraded data, we find that peaks are at similar locations but the power is lower in the degraded data, particularly in the higher frequencies. To understand the underlying cause of this frequency-dependent behavior, we analyzed coherence between time series at a fixed location with its neighboring pixels. The coherence between two time series is defined as the wavelet cross-spectrum normalized with respect 

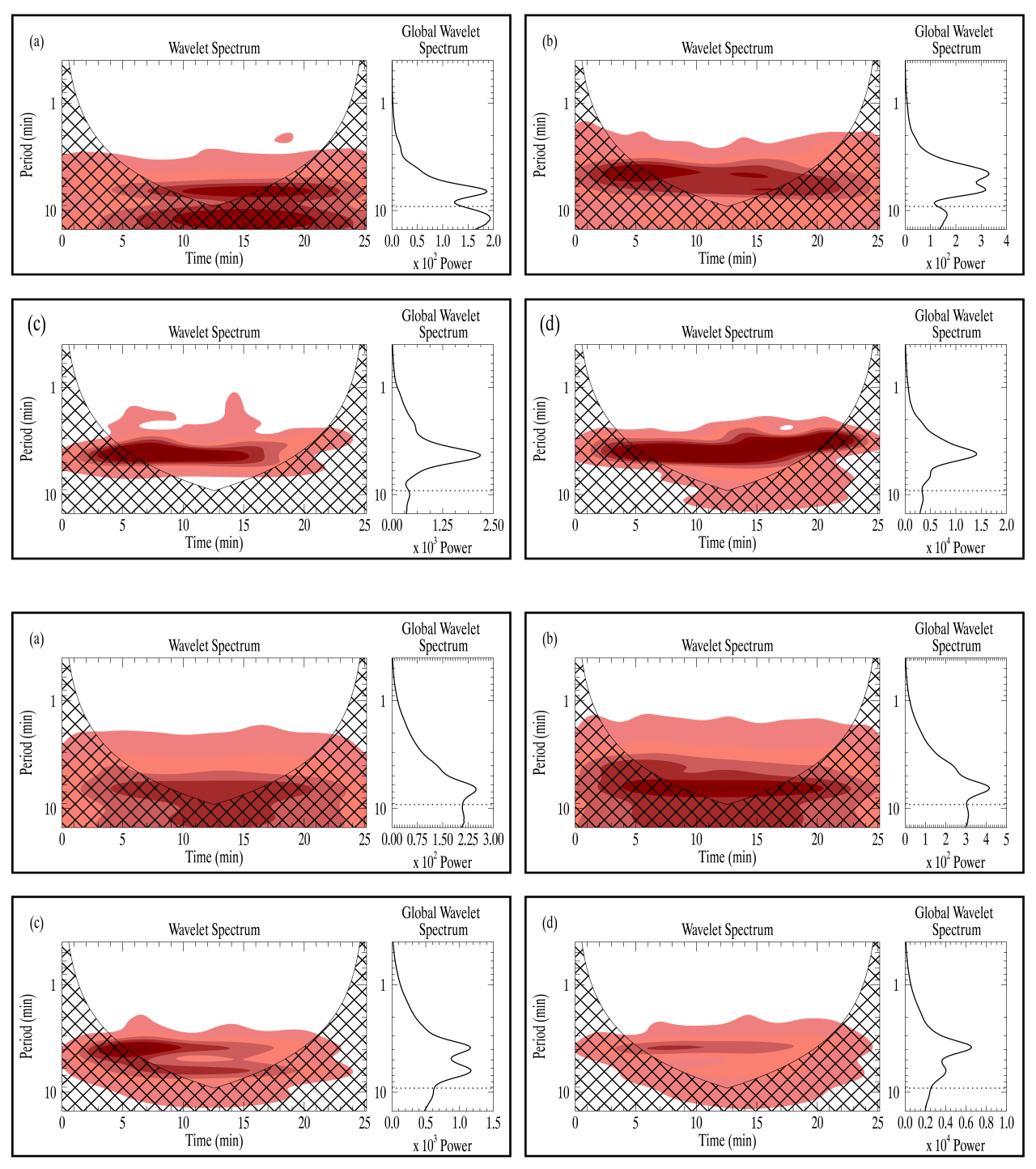

Fig. 7. Same as Fig. 6, but without the panels displaying the wave trains and averaged over all the field lines initially starting from the seed points shown as asterisks in Fig. 1 (darker color corresponds to higher power).

Fig. 8. Same as Fig. 6, but without the panels displaying the wave trains and averaged over the whole horizontal domain (darker color corresponds to higher power). to the wavelet spectrum of individual time series. It is a measure of the correlation, as a function of frequency, between two time series of the vertical velocity at two locations. We find that coherence between two nearby pixels decreases with increasing frequency. For the purposes of illustration, in Fig. 10 we show 2D maps of coherence (averaged over a frequency range) between the time series at the initial seed location (shown by the cyan asterisk in Fig. 1) and at its surrounding pixels. Here rows (a-d) correspond to the same heights for which velocity power spectra were shown in the Fig. 9. The left, middle, and right columns correspond to average coherence in the frequency range of 2-5 mHz, 6-10 mHz, and 20-25 $\mathrm{mHz}$, respectively. The selected seed point lies at the center of the maps, where the coherence is unity. Comparing the three columns we can see that spatial coherence is higher for the lower frequency range relative to the high frequency range. This implies that high frequency waves get out of phase over smaller horizontal distances compared to lowfrequency waves. This means that observations, even those with a relatively high spatial resolution of $100-200 \mathrm{~km}$, miss a significant amount of power at high frequencies. Therefore, highfrequency waves are more affected by the smearing of data as seen in Fig. 9. For instance, in the photosphere (at a height of $250 \mathrm{~km})$, the loss in power at the peak frequency $(\sim 2.5 \mathrm{mHz})$ is only $30 \%$, while at $15 \mathrm{mHz}$ only $5 \%$ of the actual power will be observed at a resolution of $100 \mathrm{~km}$. Also, in comparing different rows of Fig. 10, we can see that the spatial extent of coherence increases with height due to expansion of magnetic fields with height. It explains the comparatively smaller drop due to smearing at high frequencies at greater heights (Fig. 9). For instance, at a height of $1500 \mathrm{~km}$, observations with $100 \mathrm{~km}$ resolution would lower the power at the peak frequency $(\sim 4.5 \mathrm{mHz})$ by $20 \%$, while at $15 \mathrm{mHz} \sim 35 \%$ of the actual power will be observed.

To investigate the energy contribution of slow magnetoacoustic waves to the chromosphere, we calculate the acoustic energy flux using velocity power spectra, namely, $F_{\text {ac,tot }}\left(v_{i}\right)=$ $\rho \sum_{i} P_{v}\left(v_{i}\right) c_{s}$ (Canfield \& Musman 1973; Bello González et al. 2009) and display it as a function of height in Fig. 11. The height range is shown only up to $800 \mathrm{~km}$ for the following reasons. Plasma down-flows resulting from the artificial closed upper boundary condition can produce non-physical results above this height. Moreover, the relationship used to calculate the acoustic energy flux is valid only for linear waves and magneto-acoustic waves become nonlinear in nature approximately above this height. Wave energy flux decreases with height due to the combined effects of wave-reflection, mode-conversion, and dissipation. Since the atmospheric cutoff frequency in facular regions is reduced to $\sim 2 \mathrm{mHz}$ (Centeno et al. 2009), we integrate the velocity power over frequencies larger than $2 \mathrm{mHz}$ for the plage region 


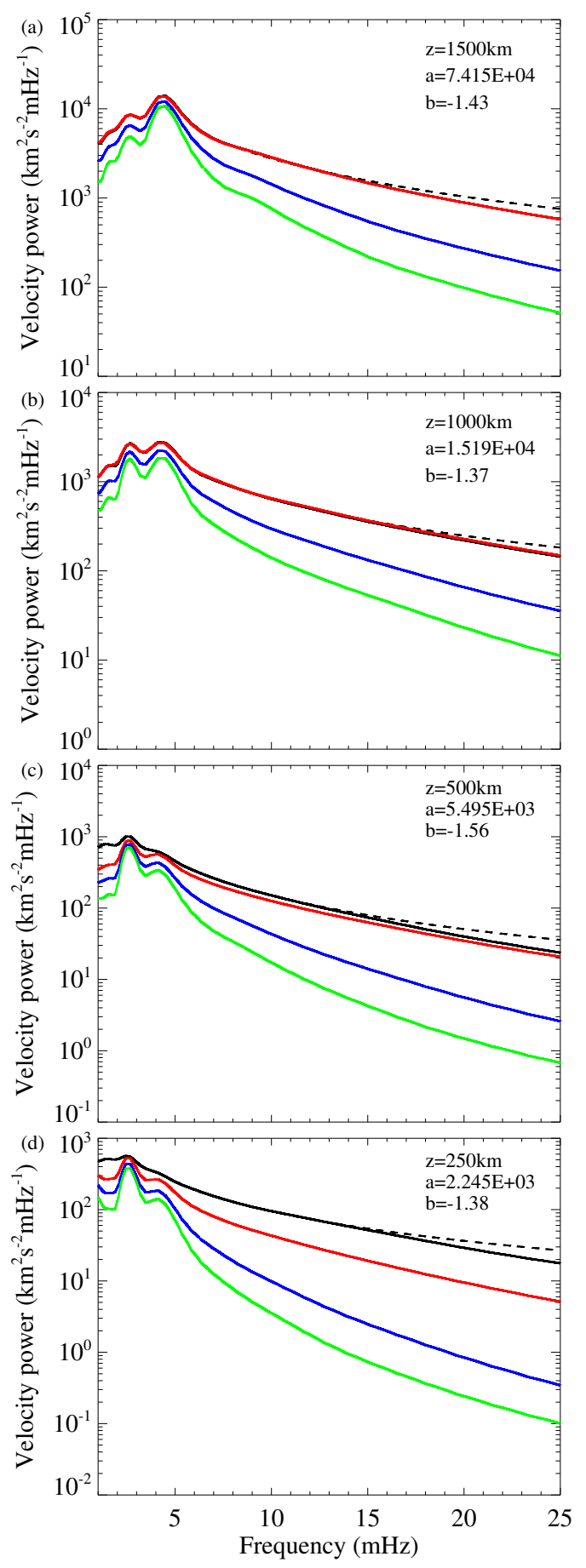

Fig. 9. Power spectra of longitudinal velocity, i.e., velocity parallel to the field (black) and vertical velocity (red) at the full resolution of simulations. Power spectra of vertical velocity for degraded simulation data with an effective resolution of $100 \mathrm{~km}$ (blue) and $200 \mathrm{~km}$ (green). Dashed curve displays a power-law fit to the black curve in the frequency range $6-25 \mathrm{mHz}$ in each panel. Different panels correspond to different heights as mentioned in each panel. Here, "a" and "b" are the amplitude and the exponent for the power law.

studied here. The height variation of acoustic wave energy flux accounting for oscillations with frequencies higher than $2 \mathrm{mHz}$ is shown in the left panel of Fig. 11. We find higher energy flux when using the parallel component of the velocity compared to the vertical component in the near-surface layers, but the two become very similar in the chromosphere as magnetic field lines are more or less vertical above $500-600 \mathrm{~km}$ for unipolar plage with $200 \mathrm{G}$ average field. Not surprisingly, degrading the spatial resolution reduces the estimated wave energy flux by a factor of 2-3 for the two resolutions tested here.

The height range from $\sim 500 \mathrm{~km}$ to $\sim 800 \mathrm{~km}$ falls in the lower chromosphere as can be seen from Fig. 8(e) of Yadav et al. (2021), which displays the mean temperature stratification for the same simulation setup. Therefore, the energy flux available at a height of $800 \mathrm{~km}$ (i.e., $\sim 10^{4} \mathrm{~W} \mathrm{~m}^{-2}$ ) is just sufficient to balance the estimated radiative losses in the layers above this height, as estimated by Withbroe \& Noyes (1977). This is in contrast to previous studies where acoustic flux was found to be insufficient to compensate for the radiative losses (Fossum \& Carlsson 2005b; Beck et al. 2009; Abbasvand et al. 2020, 2021). Additionally, the acoustic wave flux in the height range of $250-600 \mathrm{~km}$ is much larger than estimated in previous studies pertaining to quiet Sun regions (Wunnenberg et al. 2002; Straus et al. 2008; Bello González et al. 2009). This is due to two reasons: Firstly, the high spatial and temporal resolution of our simulations which efficiently capture high-frequency oscillations that are missed in lower resolution observations. Secondly, facular regions (plage) allow the propagation of low frequency waves along the many inclined field lines near the edges of magnetic elements, so that these waves can reach upper chromosphere.

To compare our results with the acoustic wave energy flux in the literature in reference to the quiet Sun, we applied a highpass filter at the atmospheric cutoff frequency in the quiet Sun of $\sim 5 \mathrm{mHz}$. The height variation of acoustic wave energy flux accounting solely for oscillations with frequencies more than $5 \mathrm{mHz}$ is shown in the right panel of Fig. 11. High frequency waves are more influenced by the spatial degradation because they have a smaller spatial coherence, as shown in Fig. 10, and are only partially captured in the spatially degraded data. Therefore, including the energy contribution only from the high frequency waves shows that degrading the resolution reduces the estimated wave energy flux by a factor of 4-6. The values for acoustic wave energy flux for degraded data (above $5 \mathrm{mHz}$ ) are similar to previously reported values for quiet Sun regions. For example, at a height of $600 \mathrm{~km}$ above surface, Wunnenberg et al. (2002) estimated an acoustic flux of $3.6 \times 10^{3} \mathrm{~W} \mathrm{~m}^{-2}$ into the non-magnetic chromosphere. Similar values were reported by Bello González et al. (2009) and Straus et al. (2008). We also obtained similar values at these heights for a degraded resolution of $\sim 200 \mathrm{~km}$ when including waves with frequencies higher than $5 \mathrm{mHz}$. However, in the case of facular regions, the cutoff frequency is $\sim 2 \mathrm{mHz}$ and thus allows low-frequency waves to propagate through the chromosphere, which contribute significantly to the total acoustic flux (Lites et al. 1993; Centeno et al. 2009; de Wijn et al. 2009; Rajaguru et al. 2019).

With this numerical experiment, we conclude that in the upper photosphere and the lower chromosphere, the acoustic energy flux is under-estimated in the observations due to poor resolution as well as due to not measuring the longitudinal component of velocity. For example, at a height of $200 \mathrm{~km}$ above the mean surface, the energy flux computed using the vertical component of velocity at full resolution is $10^{6} \mathrm{~W} \mathrm{~m}^{-2}$, which is a factor of $\sim 2$ higher than the energy flux calculated using degraded data at an effective resolution of $200 \mathrm{~km}\left(\sim 5 \times 10^{5} \mathrm{~W} \mathrm{~m}^{-2}\right)$. Moreover, the energy flux computed using the component of velocity parallel to the field at this height is $\sim 1.7 \times 10^{6} \mathrm{~W} \mathrm{~m}^{-2}$ which is another factor of $\sim 1.7$ higher calculated using the vertical component of velocity at full resolution. This means that observations at solar disc center with $200 \mathrm{~km}$ resolution are likely to catch less than a third of the total acoustic energy 

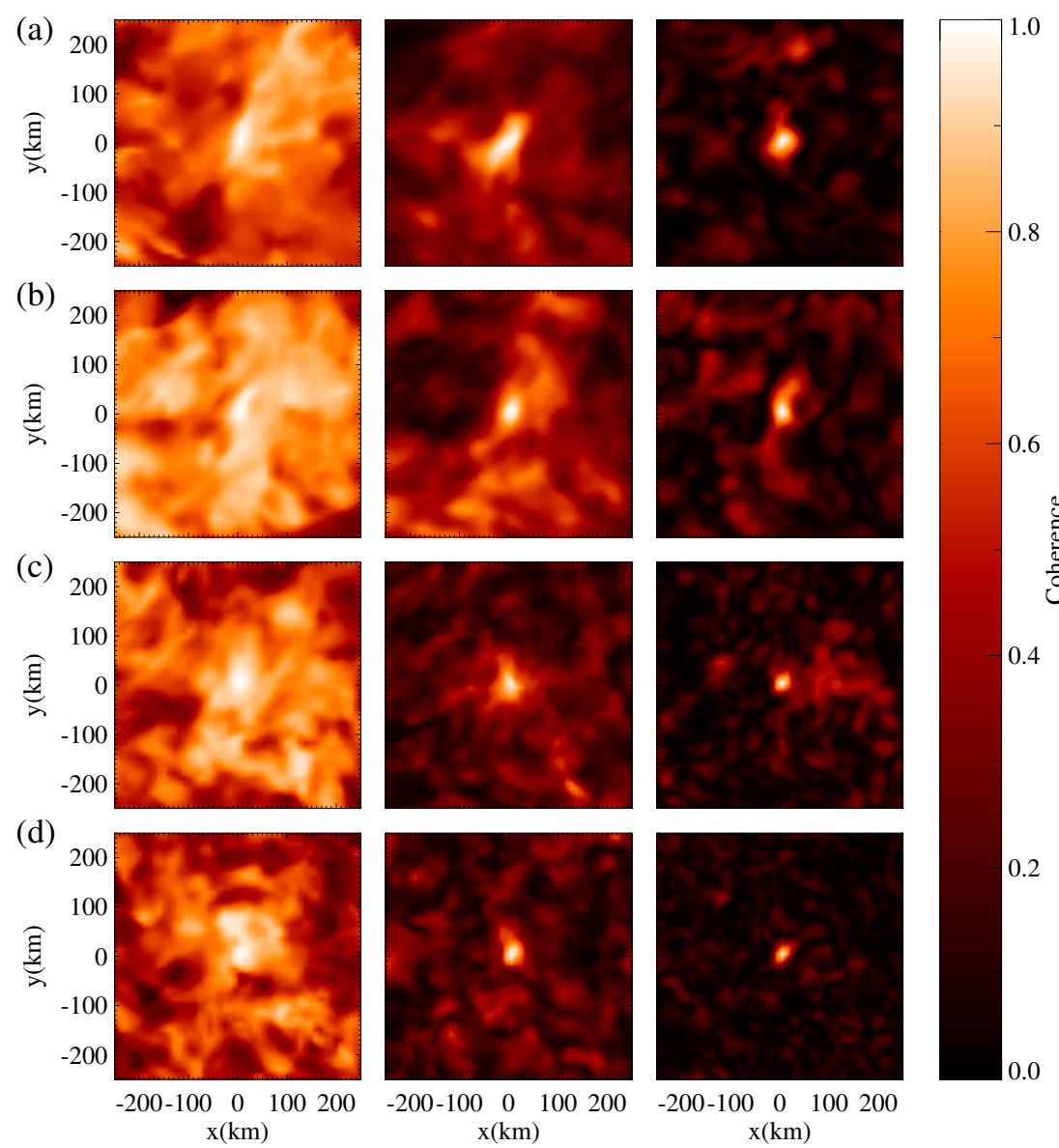

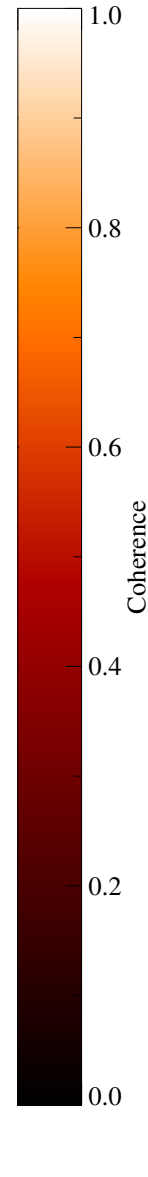

Fig. 10. 2D maps of coherence between the time series at the initial seed location (identified by the cyan asterisk in Fig. 1) and at surrounding locations at different heights viz. (a) $1500 \mathrm{~km},(b) 1000 \mathrm{~km},(c)$ $500 \mathrm{~km}$ and $(d) 250 \mathrm{~km}$ above the mean solar surface. The plotted region corresponds to the dashed square in the rightmost panel of Fig. 1. Left, middle, and right columns correspond to average coherence in the frequency range of $2-5 \mathrm{mHz}, 6-10 \mathrm{mHz}, 20-25 \mathrm{mHz}$, respectively. flux in the photosphere. We also find that the acoustic wave energy flux available in the lower and the middle chromosphere is $\sim 10^{4} \mathrm{~W} \mathrm{~m}^{-2}$, which is just sufficient to balance the estimated energy requirement in active regions in these layers that is also $\sim 10^{4} \mathrm{~W} \mathrm{~m}^{-2}$ (Vernazza et al. 1981; Withbroe \& Noyes 1977).

\section{Summary and conclusions}

In this paper, we investigate the propagation of slow magnetoacoustic waves along magnetic field lines using high-resolution $(10 \mathrm{~km})$, high-cadence $(1 \mathrm{~s}) 3 \mathrm{D}$ radiation-MHD simulations of a plage region. We find that the magnetic field lines carrying slow magneto-acoustic waves are continuously advected by the plasma flows in the photosphere. Therefore, it is important to follow the individual field lines to study properties of the waves propagating along them. Height-time maps of longitudinal velocity along the field lines show clear signatures of propagating slow magneto-acoustic waves. We applied a wavelet transform to compute the frequency spectra at various heights. The dominant frequency of slow-mode oscillations largely depends on the magnetic geometry of the region. Power spectra of longitudinal velocity averaged over the selected seed points in the core of a magnetic concentration show that the peak frequency shifts towards higher frequencies and there is negligible power found around $3 \mathrm{mHz}$ (possibly due to acoustic cut-off) as we move to higher atmospheric layers. These selected seed points, however, are associated with mostly vertical magnetic field lines representative of the central parts of magnetic concentrations. In contrast, there is significant power at frequencies around $3 \mathrm{mHz}$ in horizontally-averaged power spectra over the whole domain. This is likely due to leakage of low- frequency waves along the inclined magnetic field lines found at the edges of $\mathrm{kG}$ magnetic features, as previously suggested by de Wijn et al. (2009).

To estimate the energy contribution from high-frequency $(f>6 \mathrm{mHz})$ waves, we calculated power spectra extending up to $25 \mathrm{mHz}$. We find that power spectra in the high-frequency range $(6-25 \mathrm{mHz})$ can be approximated by a power-law of the form $a f^{-b}$ (with exponent $b$ lying around 1.5), suggesting wave-excitation due to turbulence. This is consistent with previous studies where acoustic energy generation is strongly linked to the Kolmogorov turbulent energy spectrum (Musielak et al. 1994; Ulmschneider et al. 1996). Integrating velocity power over frequency range $2-50 \mathrm{mHz}$ we find that magneto-acoustic waves carry just sufficient energy to heat the chromosphere in solar plage regions. This is in contrast to the claims by Fossum \& Carlsson (2005a) and recent observations by Abbasvand et al. (2020).

The reason for this discrepancy may have to do with our finding that at frequencies above $5 \mathrm{mHz}$ the waves are not in phase within a single magnetic flux concentration (they get out of phase even within a few tens of $\mathrm{km}$ of horizontal distance, that is, not much more than the resolution of the simulations). This has multiple important consequences. For instance, even high-resolution observations that resolve a magnetic feature (but not its sub-structure) would strongly underestimate the wave power. Also, if the wave is out of phase on field lines that are close to each other, it implies the presence of strong horizontal gradients of velocity, temperature, etc., especially in the chromosphere where the wave amplitude is large. Such gradients could lead to additional dissipation and heating (phase mixing). 
N. Yadav et al.: Slow magneto-acoustic waves in the plage chromosphere
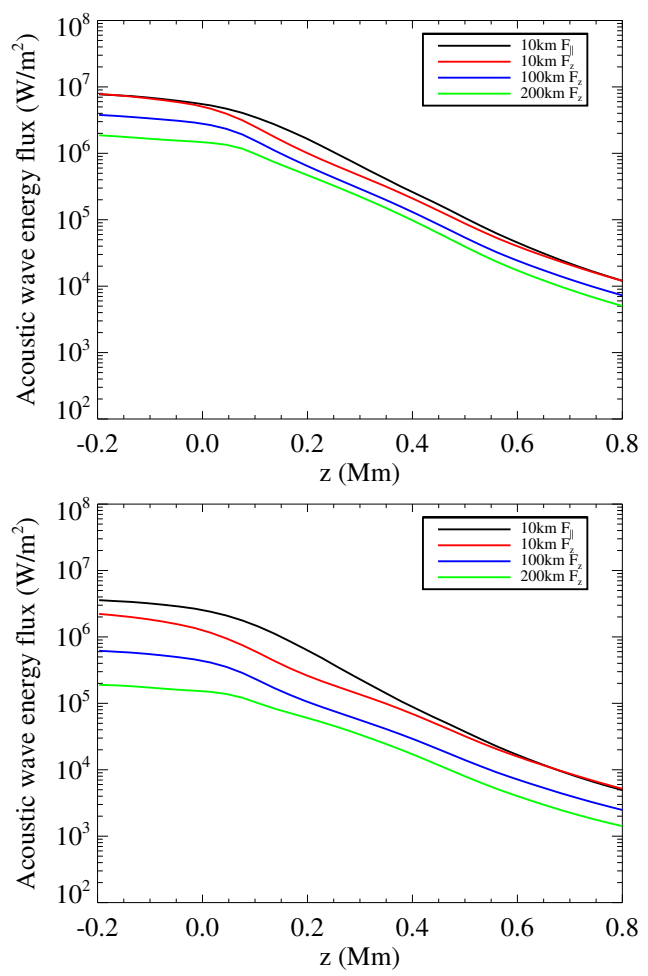

Fig. 11. Variation of acoustic wave energy flux with geometrical height computed using the component of velocity parallel to the magnetic field for full resolution (black), computed using the vertical component of the velocity for full resolution (red), computed using the vertical component of the velocity for degraded data at an effective resolution of $100 \mathrm{~km}$ (blue) and $200 \mathrm{~km}$ (green). Left (Right): integrating velocity power spectra within the frequency range $2-500 \mathrm{mHz}(5-500 \mathrm{mHz})$.

To test the first of these consequences, we additionally degraded our simulation data to effective resolutions of $100 \mathrm{~km}$ and $200 \mathrm{~km}$, and compared the total energy flux in the degraded data with simulation data at full resolution. We find that even at a high spatial resolution of $100 \mathrm{~km}$, observations may strongly underestimate the wave energy flux. The observations may also miss some of the flux due to the fact that only the lineof-sight velocity is measured and not the velocity along the magnetic field line, which determines the energy flux carried by the wave. Together, these two effects may lead to an underestimate of the energy flux by up to a factor of three in the upper photosphere and the lower chromosphere deduced from observations at spatial resolution of $200 \mathrm{~km}$. At lower spatial resolutions, even more of the wave energy flux will be missed. For instance, Fossum \& Carlsson (2005a) found that wave energy flux of highfrequency acoustic waves to be too low, by a factor of at least 10, to balance the radiative losses in the solar chromosphere. This could be due to the coarse spatial sampling $\left(\sim 0.5^{\prime \prime}\right.$ pixel $\left.^{-1}\right)$ of the instrument and large width of the response heights of the observed passbands.

It is important to note that although the new generation of large telescopes - foremost among them the Daniel K. Inoue Solar Telescope (DKIST) but in the future, the European Solar Telescope (EST) as well - will hopefully largely overcome the loss in wave energy due to limited spatial resolution of observations, they will likely still miss more than half of the longitudinal wave energy flux in plages due to the fact that only the line-of-sight component of the velocity field is measured. In quiet Sun regions, we expect observations to miss an even larger fraction of the longitudinal wave energy flux, as magnetic features expand faster and the difference between the line-of-sight velocity and the actual longitudinal velocity of the wave is greater.

Acknowledgements. The authors thank L. P. Chitta and D. Przyblski for useful discussions on various aspects of this paper. This project has received funding from the European Research Council (ERC) under the European Union's Horizon 2020 research and innovation programme (grant agreement No. 695075) and has been supported by the BK21 plus program through the National Research Foundation (NRF) funded by the Ministry of Education of Korea.

\section{References}

Abbasvand, V., Sobotka, M., Heinzel, P., et al. 2020, ApJ, 890, 22 Abbasvand, V., Sobotka, M., Švanda, M., et al. 2021, A\&A, 648, A28 Barthol, P., Gandorfer, A., Solanki, S. K., et al. 2011, Sol. Phys., 268, 1 Beck, C., Khomenko, E., Rezaei, R., \& Collados, M. 2009, A\&A, 507, 453 Bel, N., \& Leroy, B. 1977, A\&A, 55, 239

Bello González, N., Flores Soriano, M., Kneer, F., \& Okunev, O. 2009, A\&A, 508, 941

Bello González, N., Flores Soriano, M., Kneer, F., Okunev, O., \& Shchukina, N. 2010a, A\&A, 522, A31

Bello González, N., Franz, M., Martínez Pillet, V., et al. 2010b, ApJ, 723, L134 Berkefeld, T., Schmidt, W., Soltau, D., et al. 2011, Sol. Phys., 268, 103 Bhatnagar, A., \& Tanaka, K. 1972, Sol. Phys., 24, 87

Biermann, L. 1948, Z. Astrophys., 25, 161

Bogdan, T. J. 2000, Sol. Phys., 192, 373

Bogdan, T. J., \& Judge, P. G. 2006, Phil. Trans. R. Soc. London, Ser. A, 364, 313 Buehler, D., Lagg, A., van Noort, M., \& Solanki, S. K. 2019, A\&A, 630, A86

Cally, P. S. 2007, Astron. Nachr., 328, 286

Canfield, R. C., \& Musman, S. 1973, ApJ, 184, L131

Cavallini, F. 2006, Sol. Phys., 236, 415

Centeno, R., Collados, M., \& Trujillo Bueno, J. 2006, ApJ, 640, 1153

Centeno, R., Collados, M., \& Bueno, J. T. 2009, ApJ, 692, 1211

Chae, J., Lee, J., Cho, K., et al. 2017, ApJ, 836, 18

Chandrasekhar, S. 1957, An Introduction to the Study of Stellar Structure

Chelpanov, A. A., Kobanov, N. I., \& Kolobov, D. Y. 2016, Sol. Phys., 291, 3329

Cheung, C. 2006, PhD Dissertation, Georg-August-Universität zu Göttingen, Germany

Chitta, L. P., van Ballegooijen, A. A., Rouppe van der Voort, L., DeLuca, E. E., \& Kariyappa, R. 2012, ApJ, 752, 48

Clyne, J., \& Rast, M. 2005, Proc. SPIE-IS\&T Electronic Imaging, 5669, 284

Clyne, J., Mininni, P., Norton, A., \& Rast, M. 2007, New J. Phys., 9, 301

Cuntz, M., Rammacher, W., \& Musielak, Z. E. 2007, ApJ, 657, L57

De Pontieu, B., Erdélyi, R., \& James, S. P. 2004, Nature, 430, 536

De Pontieu, B., Erdélyi, R., \& Moortel, I. D. 2005, ApJ, 624, L61

De Pontieu, B., Hansteen, V. H., Rouppe van der Voort, L., van Noort, M., \& Carlsson, M. 2007, ApJ, 655, 624

de Wijn, A. G., McIntosh, S. W., \& Pontieu, B. D. 2009, ApJ, 702, L168

Felipe, T., \& Sangeetha, C. R. 2020, A\&A, 640, A4

Fleck, B., \& Schmitz, F. 1991, A\&A, 250, 235

Fossum, A., \& Carlsson, M. 2005a, Nature, 435, 919

Fossum, A., \& Carlsson, M. 2005b, ApJ, 625, 556

Fossum, A., \& Carlsson, M. 2006, ApJ, 646, 579

Gandorfer, A., Grauf, B., Barthol, P., et al. 2011, Sol. Phys., 268, 35

Goldreich, P., \& Keeley, D. A. 1977, ApJ, 212, 243

Handy, B., Acton, L., Kankelborg, C., et al. 1999, Sol. Phys., 187, 229

Hansteen, V. H., De Pontieu, B., Rouppe van der Voort, L., van Noort, M., \& Carlsson, M. 2006, ApJ, 647, L73

Hasan, S. S., Kalkofen, W., \& van Ballegooijen, A. A. 2000, ApJ, 535, L67

Hasan, S. S., van Ballegooijen, A. A., Kalkofen, W., \& Steiner, O. 2005, ApJ, 631,1270

Jafarzadeh, S., Solanki, S. K., Feller, A., et al. 2013, A\&A, 549, A116

Jafarzadeh, S., Solanki, S. K., Stangalini, M., et al. 2017, ApJS, 229, 10

Jess, D. B., Andić, A., Mathioudakis, M., Bloomfield, D. S., \& Keenan, F. P. 2007, A\&A, 473, 943

Kalkofen, W. 2007, ApJ, 671, 2154

Kato, Y., Steiner, O., Steffen, M., \& Suematsu, Y. 2011, ApJ, 730, L24

Kato, Y., Steiner, O., Hansteen, V., et al. 2016, ApJ, 827, 7

Khomenko, E., \& Cally, P. S. 2012, ApJ, 746, 68

Khomenko, E., \& Collados, M. 2015, Liv. Rev. Sol. Phys., 12, 6

Khomenko, E., Centeno, R., Collados, M., \& Bueno, J. T. 2008, ApJ, 676, L85

Kostik, R., \& Khomenko, E. 2013, A\&A, 559, A107

Lawrence, J. K., Cadavid, A. C., Christian, D. J., Jess, D. B., \& Mathioudakis, M. 2011, ApJ, 743, L24 
Lites, B. W. 1986, ApJ, 301, 992

Lites, B. W., \& Thomas, J. H. 1985, ApJ, 294, 682

Lites, B. W., Rutten, R. J., \& Kalkofen, W. 1993, ApJ, 414, 345

Mathioudakis, M., Jess, D. B., \& Erdélyi, R. 2013, Space Sci. Rev., 175, 1

McIntosh, S. W., \& Jefferies, S. M. 2006, ApJ, 647, L77

Michalitsanos, A. G. 1973, Sol. Phys., 30, 47

Musielak, Z. E., Rosner, R., Stein, R. F., \& Ulmschneider, P. 1994, ApJ, 423, 474

Nordlund, Å., \& Stein, R. F. 2001, ApJ, 546, 576

Pietarila, A., Hirzberger, J., Zakharov, V., \& Solanki, S. K. 2009, A\&A, 502, 647

Praderie, F., \& Thomas, R. N. 1976, Sol. Phys., 50, 333

Rajaguru, S. P., Sangeetha, C. R., \& Tripathi, D. 2019, ApJ, 871, 155

Reardon, K. P., Lepreti, F., Carbone, V., \& Vecchio, A. 2008, ApJ, 683, L207

Rempel, M. 2014, ApJ, 789, 132

Rempel, M. 2017, ApJ, 834, 10

Schwartz, S. J., \& Bel, N. 1984, A\&A, 137, 128

Schwarzschild, M. 1948, ApJ, 107, 1

Solanki, S. K., Inhester, B., \& Schüssler, M. 2006, Rep. Prog. Phys., 69, 563

Solanki, S. K., Barthol, P., Danilovic, S., et al. 2010, ApJ, 723, L127
Straus, T., Fleck, B., Jefferies, S. M., et al. 2008, ApJ, 681, L125

Torrence, C., \& Compo, G. P. 1998, Bull. Am. Meteorol. Soc., 79, 61

Ulmschneider, P., Theurer, J., \& Musielak, Z. E. 1996, A\&A, 315, 212

Ulmschneider, P., Rammacher, W., Musielak, Z. E., \& Kalkofen, W. 2005, ApJ, 631, L155

van Ballegooijen, A. A., Asgari-Targhi, M., Cranmer, S. R., \& DeLuca, E. E. 2011, ApJ, 736, 3

Vecchio, A., Cauzzi, G., \& Reardon, K. P. 2009, A\&A, 494, 269

Vernazza, J. E., Avrett, E. H., \& Loeser, R. 1981, ApJS, 45, 635

Verth, G., \& Jess, D. B. 2016, MHD Wave Modes Resolved in Fine-Scale Chromospheric Magnetic Structures (American Geophysical Union (AGU)), 431

Vigeesh, G., Fedun, V., Hasan, S. S., \& Erdélyi, R. 2012, ApJ, 755, 18

Vögler, A., Shelyag, S., Schússler, M., et al. 2005, A\&A, 429, 335

Withbroe, G. L. 1983, ApJ, 267, 825

Withbroe, G. L., \& Noyes, R. W. 1977, ARA\&A, 15, 363

Wunnenberg, M., Kneer, F., \& Hirzberger, J. 2002, A\&A, 395, L51

Yadav, N., Cameron, R. H., \& Solanki, S. K. 2020, ApJ, 894, L17

Yadav, N., Cameron, R. H., \& Solanki, S. K. 2021, A\&A, 645, A3 